\title{
Novel Copper(II) Induced Formation of a Porphyrinogen Derivative: X-ray Structural, Spectroscopic, and Electrochemical Studies of Porphyrinogen Complexes of $\mathrm{Cu}$ (II) and $\mathrm{Co}$ (III) Complex of a Trispyrazolyl Tripodal Ligand
}

\author{
Sachindranath Paul, ${ }^{\dagger}$ Anil Kumar Barik, ${ }^{\ddagger}$ Shie Ming Peng, ${ }^{\S}$ and Susanta Kumar Kar ${ }^{*}, \|$ \\ Department of Chemistry, SreeChaitanya College, Habra, North 24 Parganas, West Bengal, India, \\ Department of Chemistry, St. Paul's C.M. College, 33/1, Raja Rammohan Roy Sarani, \\ Calcutta 700 009, India, Department of Chemistry, National Taiwan University, Taipei, \\ Taiwan, 106 Republic of China, and Department of Chemistry, University College of Science, \\ 92 A.P.C. Road, Calcutta 700 009, India
}

Received November 29, 2001

Copper(II) complexes of a novel pyrazole containing porphyrinogen and cobalt(III) and zinc(II) complexes of a pyrazole containing tripodal ligand having $\mathrm{N}$-donor atoms have been investigated. 5-Methyl-3-formylpyrazole (MPA) on reaction with copper(II) nitrate or perchlorate in the presence of tris(2-aminoethyl)amine (tren) forms novel pyrazole-based porphyrinogen complexes $\left[\mathrm{Cu}\left(\mathrm{T}_{3}\right.\right.$-porphyrinogen $\left.)\left(\mathrm{H}_{2} \mathrm{O}\right)\right]\left(\mathrm{NO}_{3}\right)_{2}(1 \mathrm{a})$ and $\left[\mathrm{Cu}\left(\mathrm{T}_{3}\right.\right.$-porphyrinogen $\left.)\left(\mathrm{H}_{2} \mathrm{O}\right)\right]-$ $\left(\mathrm{ClO}_{4}\right)_{2}$ (1b) where $\mathrm{T}_{3}$-porphyrinogen is 1,6,11,16-tetraaza-5,10,15,20-tetrahydroxy-2,7,12,17-tetramethylporphyrinogen. The same products are also obtained when tren is replaced by triethylamine. By contrast, the reaction between MPA, tren, and cobalt(II) perchlorate produces the cobalt(III) complex $\left[\mathrm{Co}\left(\mathrm{HMPz}_{3}\right.\right.$ tren) $] \mathrm{ClO}_{4}$ (2) derived from the tripodal Schiff base tris[4-(3-(5-methyl-pyrazolyl)-3-aza-3-butenyl]amine $\left(\mathrm{H}_{3} \mathrm{MPz}_{3}\right.$ tren). The $\mathrm{X}$-ray crystal structures of the copper(II) complexes (1a and $\mathbf{1 b}$ ) and the cobalt(III) complex (2) have been determined. The structures show distorted square pyramidal coordination environments for $\mathbf{1 a}$ and $\mathbf{1} \mathbf{b}$ with the water molecule occupying the apical site, while for complex 2 a distorted octahedral geometry is obtained. Data for 1a follow: $a=19.476(3) \AA$, $b=9.4116(8) \AA, c=14.204(3) \AA ; \alpha=90^{\circ}=\gamma, \beta=107.58(2)^{\circ} ; V=2482.0(7) \AA^{3}, Z=4$. Data for 1b follow: $a=20.967(3) \AA, b=9.1563(18) \AA, c=14.858(4) \AA ; \alpha=90^{\circ}=\gamma, \beta=108.44(3)^{\circ} ; V=2706.0(10) \AA^{3}, Z=$ 4. Data for 2 follow: $a=21.293(3) \AA, b=12.724(2) \AA, c=19.777(4) \AA ; \alpha=90^{\circ}=\gamma, \beta=93.03(2)^{\circ} ; V=$ $5350.6(15) \AA^{3}, Z=8$. All three complexes crystallize in the monoclinic crystal system with the $C 2 / c$ space group. The complexes are further characterized by UV-vis, IR, EPR, and electrochemical studies.

\section{Introduction}

Metal complexes of chiral porphyrins, inverted porphyrins, porphyrin oligomers, and metalloporphyrins are of great current interest because of their relevance to asymmetric synthesis, chiral recognition, stabilization of unusual oxidation states, and the mechanism of photosynthesis. ${ }^{1-5}$ Porphyrinogen is the chemical and biochemical precursor of porphyrins. ${ }^{6}$ Bonomo et al. in a recent study have reported the genesis of porphodimethene, the skeleton paving the way

* To whom correspondence should be addressed. E-mail: skkar@ mail.cucc.ernet.in.

$\dagger$ SreeChaitanya College.

St. Paul’s C.M. College.

$\S$ National Taiwan University.

" University College of Science.

10.1021/ic0112185 CCC: $\$ 22.00$ Published on Web 10/08/2002 from porphyrinogen to porphyrin. ${ }^{7}$ Several recent studies have focused on the development of core-modified porphyrins and their metalation. ${ }^{1-8}$ Moreover, large numbers of

(1) Latos-Grazynski, L. Core Modified Heteroanalogues of Porphyrins and Metalloporphyrins. In The Porphyrin Handbook; Kadish, K. M., Smith, K. M., Guilard, R., Eds.; Academic Press: New York, 2000; Vol. 2, p 361.

(2) Mazzanti, M.; Marchon, J.-C.; Wojaczynski, J.; Wolowiec, S.; LatosGrazynski, L.; Shang, M.; Scheidt, W. R. Inorg. Chem. 1998, 37, 2476-2481.

(3) Chmielewski, P. J.; Latos-Grazynski, L.; Schmidt, I. Inorg. Chem. 2000, 39, 5475-5482 and references therein.

(4) Wojaczynski, J.; Latos-Grazynski, L.; Chmielewski, P. J.; Calcar, P. V.; Balch, A. L. Inorg. Chem. 1999, 38, 3040-3050.

(5) (a) Floriani, C.; Floriani-Moro, R. In The Porphyrin Handbook; Kadish, K. M., Smith, K. M., Guilard, R., Eds.; Academic: Burlington, MA, 1999; Vol. 3, Chapter 25 and references therein. (b) Bonomo, L.; Solari, E.; Floriani, C.; Chiesi-villa, A.; Rizzoli, C. J. Am. Chem. Soc. 1998, 120, 12972.

Inorganic Chemistry, Vol. 41, No. 22, 20025803 
Paul et al.

metalloproteins are involved in different biological electrontransfer reactions as well as in redox processes involving molecular oxygen where the $\mathrm{Cu}(\mathrm{II})$ ion is found in active sites. $^{9-15}$ The coordination chemistry of tripodal ligands has received considerable attention in recent years because of the diversity of physicochemical and structural features displayed by their metal complexes. ${ }^{16-21}$ Some of these ligands have been used to mimic the active sites of several metalloproteins. For example, copper complexes of sterically demanding tris(pyrazolyl) hydroborate ligands have found application as synthetic analogues for cuproprotein active sites. ${ }^{22}$ Hemocyanine (HC), ${ }^{13,23}$ the most effective model to date for the dioxygen transport protein, utilizes the ligand tris[1,3,5-diisopropylpyrazole] hydroborate.

Attempts have been made to prepare $\mathrm{Cu}(\mathrm{II}), \mathrm{Co}(\mathrm{III})$, and $\mathrm{Zn}(\mathrm{II})$ complexes of the tripodal ligand $\mathrm{H}_{3} \mathrm{MPz}_{3}$ tren by the reaction of tren and $\mathrm{MPA}^{24}$ in 1:3 molar proportion and corresponding metal salts in ethanol solution using template methodology. In the case of $\mathrm{Cu}$ complexes, we have a novel observation. The $\mathrm{Cu}(\mathrm{II})$ ion induced macrocyclic complexes are isolated instead of those with the desired tripodal ligand. Thus, tren acts as a base in $\mathrm{Cu}(\mathrm{II})$ ion induced macrocycle

(6) (a) Porphyrins and Metalloporphyrins; Smith, K. M., Ed.; Elsevier: Amsterdam, 1975. The Porphyrins; Dolphin, D., Ed.; Academic: New York, 1978. (b) Mashiko, T.; Dolphin, D. In Comprehensive Coordination Chemistry; Wilkinson, G., Gillard, R. D., McCleverty, J. A., Eds.; Pergamon: Oxford, U.K., 1987; Vol. 2, Chapter 21.1, p 855. (c) Kim, J. B.; Adler, A. D.; Longo, R. F. In The Porphyrins; Dolphin, D., Ed.; Academic: New York, 1978; Vol. 1, Part A, p 85. Mauzerall, D. In The Porphyrins; Dolphin, D., Ed.; Academic: New York, 1978; Vol. 2, p 91. (d) Lindsey, J. S.; Schreiman, I. C.; Hsu, H. C.; Kearney, P. C.; Marguerettaz, A. M. J. Org. Chem. 1987, 52, 827. Lindsey, J. S.; Wagner, R. W. J. Org. Chem. 1989, 54, 828. (e) Biosynthesis of Tetrapyrroles; Jordan, P. M., Ed.; Elsevier: New York, 1991.

(7) Bonomo, L.; Solari, E.; Scopelliti, R.; Floriani, C.; Re, N. J. Am. Chem Soc. 2000, 122, 5312-5326.

(8) (a) Pushpan, S. K.; Srinivasan, A.; Anand, V. G.; Venkatraman, S.; Chandrashekar, T. K.; Joshi, B. S.; Roy, R.; Furuta, H. J. Am. Chem. Soc. 2001, 123, 5138. (b) Pushpan, S. K.; Srinivasan, A.; Anand, V. G.; Chandrashekar, T. K.; Subramanian, A.; Roy, R.; Sugiura, K.-I.; Sakata, Y. J. Org. Chem. 2001, 66, 153.

(9) Holm, R. H.; Kennepohl, P.; Solomon, P. I. Chem. Rev. 1996, 96, 2239-2314.

(10) Karlin, K. D.; Zuberbühler, A. D. In Bioinorganic Catalysis, 2nd ed; Reedijk, J., Bouman, E., Eds.; Marcel Dekker: New York, 1999; pp 469-534.

(11) Fox, S.; Karlin, K. D. In Active Oxygen in Biochemistry; Valentine, J. S., Foote, C. S., Greenberg, A., Liebman, J. F., Eds.; Blackie Academic and Professional: Glasgow, Scotland, 1995; pp 188-231.

(12) Kaim, W.; Rall, J. Angew. Chem. 1996, 108, 47-64.

(13) Kitajima, N.; Moro-oka, Y. Chem. Rev. 1994, 94, 737-757.

(14) Kitajima, N. Adv. Inorg. Chem. 1992, 39, 1-77.

(15) Bioinorganic Chemistry of Copper; Karlin, K. D., Tycklar, Z., Eds.; Chapman \& Hall: New York, 1993.

(16) Orvig, C.; Berg. D. J.; Rettig, S. J. J. Am. Chem. Soc. 1991, 113, 2528 .

(17) Kirchner, R. M.; Mealli, C.; Bailey, M.; Howe, M.; Torre, L. P.; Wilson, L. J.; Andrews, L. C.; Rose, N. J.; Lingafelter, E. C. Coord. Chem. Rev. 1987, 77, 89.

(18) Raghunathan, K. G.; Bharadwaj, P. K. J. Chem. Soc., Dalton Trans. 1992, 2417.

(19) Ghosh, P.; Shukla, R.; Chand, D. K.; Bharadwaj, P. K. Tetrahedron 1995, 51, 3265.

(20) Sorrell, T. N.; Allen, W. E.; White, P. S. Inorg. Chem. 1995, 34, 952.

(21) Mohamadou, A.; Gérad, C. J. Chem. Soc., Dalton Trans. 2001, 3320.

(22) Trofimenko, S. Chem. Rev. 1993, 93, 943.

(23) Reviews of HC model system: (a) Sorrell, T. N. Tetrahedron 1989, 45, 3. (b) Karlin, K. D.; Tyeklar, Z. Adv. Inorg. Biochem. 1994, 9 , 120.

(24) Abbreviations used: tren, tris(2-aminoethyl)amine; MPA, 5-methyl3-formyl-pyrazole; TBAP, tetrabutylammonium perchlorate; DPPH, diphenylpicrylhydrazyl; TBP, trigonal bipyramid.
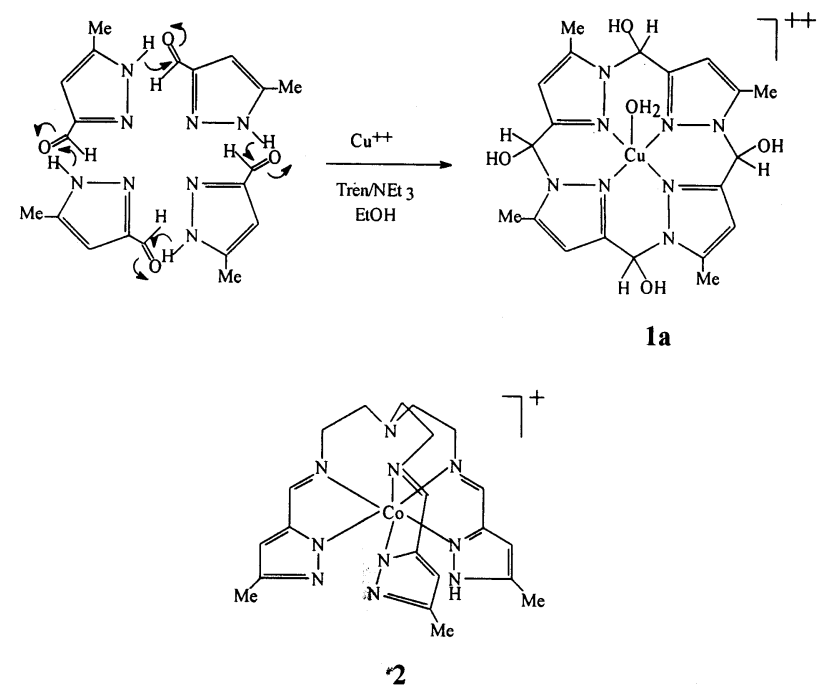

Figure 1. Plausible mechanism for macrocycle formation and structure of cation of the $\mathrm{Co}$ (III) tripodal complex.

formation, whereas it plays the pendant arm forming part of the tripodal ligand in the cases of $\mathrm{Co}(\mathrm{II})$ and $\mathrm{Zn}$ (II) ions. In this macrocycle formation, four pyrazole aldehyde units fuse together via aldol type condensation. We have also synthesized the macrocyclic complexes using equivalent amounts of another organic base, triethylamine $\left(\mathrm{NEt}_{3}\right)$.

Although various types of metal ion complexes with porphyrin or porphyrinogen-like macrocyclic ligands from pyrrole or other heterocycles ${ }^{1-8,25}$ have been synthesized and characterized, the macrocycle from pyrazole aldehyde is a new observation, and to the best of our knowledge, such type of macrocycle has not been reported until now. However, Bode et al., ${ }^{26}$ in a recent report, described the synthesis of a different type of macrocycle with built-in pyrazole groups and formation of its dinuclear $\mathrm{Cu}$ (II) complexes.

We have synthesized the Co(III) complex where the tripodal ligand formed in situ acts as a binegative $\mathrm{N}_{6}$-donor system. We have also tried to synthesize the Co(III) complex in weakly alkaline media using $1 \mathrm{mmol} / 2 \mathrm{mmol}$ organic base $\left(\mathrm{NEt}_{3}\right)$ and also in neutral medium. But interestingly, only one complex with same metal ligand stoichiometric ratio is obtained in all the cases. $\mathrm{Ni}$ (II) complexes of the same tripodal ligand have been synthesized, characterized and reported earlier. ${ }^{27}$ The plausible mechanism for the macrocycle formation and both the structures of $\mathrm{Cu}$ (II) complex of the macrocyclic ligand and $\mathrm{Co}(\mathrm{III})$ complex of the tripodal one are given in Figure 1.

\section{Experimental Section}

Materials. Tren and DMSO were purchased from Aldrich. MPA was synthesized as described earlier. ${ }^{27,28}$ Spectrograde solvents were

(25) (a) Kalis, H. R.; Latos-Grazynski, L.; Balch, A. L. J. Am. Chem. Soc. 2000, 122, 12478. (b) Wolowiec, S.; Latos-Grazynski, L.; Mazzanti, M.; Marchon, J. C. Inorg. Chem. 1997, 36, 5761. (c) Chmielewski, P. J.; Latos-Grazynski, L. Inorg. Chem. 1997, 36, 840. (d) Chmielewski, P. J.; Latos-Grazynski, L. Inorg. Chem. 2000, 39, 5639.

(26) Bode, R. H.; Bol, J. E.; Driessen, W. L.; Hulsbergen, F. B.; Reedijk, J.; Spek, A. L. Inorg. Chem. 1999, 38, 1239.

(27) Paul, S.; Barik, A. K.; Butcher, R. J.; Kar, S. K. Polyhedron 2000 $19,2661-2666$. 
used for physical measurements. All the other reagents and solvents were purchased from commercial sources and purified by standard procedures.

Syntheses. Warning! Perchlorate salts are potentially explosive and were handled only in small quantities with care.

Method A: $\left[\mathrm{Cu}\left(\mathrm{T}_{3}\right.\right.$-porphyrinogen $\left.)\left(\mathrm{H}_{2} \mathrm{O}\right)\right]\left(\mathrm{NO}_{3}\right)_{2}$ (1a). A mixture of tren $(0.146 \mathrm{~g}, 1 \mathrm{mmol})$ and MPA $(0.33 \mathrm{~g}, 3 \mathrm{mmol})$ in EtOH $(30 \mathrm{~mL})$ was refluxed for $3 \mathrm{~h}$. Then, the light yellow solution was cooled and filtered. To the filtrate was added $\mathrm{Cu}\left(\mathrm{NO}_{3}\right)_{2} \cdot 6 \mathrm{H}_{2} \mathrm{O}(0.30$ $\mathrm{g}, 1 \mathrm{mmol}$ ), and the mixture refluxed again for ca. $2 \mathrm{~h}$. The light green solution was then filtered and left in the air for slow evaporation at room temperature. A violet crystalline compound appeared after a week. It was filtered and washed with EtOH and dried in air (yield 40\%). The product was recrystallized from hot EtOH. X-ray quality violet crystals were obtained by slow evaporation of the ethanolic solution of the complex at room temperature.

Method B. A mixture of MPA $(0.44 \mathrm{~g}, 4 \mathrm{mmol})$ and $\mathrm{NEt}_{3}(0.7$ $\mathrm{mL}$ ) was refluxed in absolute EtOH $(30 \mathrm{~mL})$ for ca. $3 \mathrm{~h}$. To it was added $\mathrm{Cu}\left(\mathrm{NO}_{3}\right)_{2} \cdot 6 \mathrm{H}_{2} \mathrm{O}(0.30 \mathrm{~g}, 1 \mathrm{mmol})$, and the mixture refluxed again for $2 \mathrm{~h}$. The light green solution was then filtered and kept for slow evaporation at room temperature. After a week, a violet crystalline compound was obtained. It was filtered and washed with EtOH and air-dried (yield 45\%). Anal. Calcd for $\mathrm{C}_{20} \mathrm{H}_{26} \mathrm{CuN}_{10} \mathrm{O}_{11}$ : C, 37.15; H, 4.02; N, 21.67. Found: C, 36.98; H, 4.14; N, 21.58. IR $\left(\mathrm{KBr} ; \mathrm{cm}^{-1}\right)$ : 3365, $3228\left(v_{\mathrm{OH}}\right), 1556(\mathrm{w}, \mathrm{C}=\mathrm{NPz}), 1387(\mathrm{br}$, $\left.v_{\mathrm{NO}_{3}}^{-}\right), 1069-1032\left(\mathrm{~ms}, \nu_{\mathrm{N}-\mathrm{NPz}}\right), 383\left(v_{\mathrm{Cu}-\mathrm{O}\left(\mathrm{H}_{2} \mathrm{O}\right)}\right), 254\left(v_{\mathrm{Cu}-\mathrm{NPz} \text { ring }}\right)$. Diffuse reflectance spectroscopy (DRS) $\left(\lambda_{\max }, \mathrm{nm}\right): 566$. UV-vis (MeOH, $\lambda_{\max }, \mathrm{nm}\left(\epsilon, \mathrm{M}^{-1} \mathrm{~cm}^{-1}\right)$ ): 718 (69.9). UV-vis (DMF, $\lambda_{\max }$, $\left.\mathrm{nm}\left(\epsilon, \mathrm{M}^{-1} \mathrm{~cm}^{-1}\right)\right)$ : 787 (47). UV-vis (DMSO, $\lambda_{\max }, \mathrm{nm}\left(\epsilon, \mathrm{M}^{-1}\right.$ $\left.\mathrm{cm}^{-1}\right)$ ): 854 (52) (broad). Molar conductivity ( $\mathrm{MeOH}$ solution): $\Lambda_{\mathrm{m}}=172 \Omega^{-1} \mathrm{~cm}^{2} \mathrm{~mol}^{-1}$. EPR $\left(\mathrm{CH}_{3} \mathrm{OH} / \mathrm{C}_{2} \mathrm{H}_{5} \mathrm{OH}\right)(77 \mathrm{~K}): g_{\|}=$ 2.290, $A_{\|}=160 \mathrm{G}, g_{\perp}=2.067$. Magnetism (solid state, room temperature): $\mu_{\mathrm{eff}}=1.70 \mu_{\mathrm{B}}$.

$\left[\mathrm{Cu}\left(\mathrm{T}_{3}\right.\right.$-porphyrinogen $\left.)\left(\mathrm{H}_{2} \mathrm{O}\right)\right]\left(\mathrm{ClO}_{4}\right)_{2} \quad(\mathbf{1 b})$. This compound was prepared following any one of the procedures described for 1a, except that $\mathrm{Cu}\left(\mathrm{ClO}_{4}\right)_{2} \cdot 6 \mathrm{H}_{2} \mathrm{O}$ was used as the metal ion precursor. Yield: 40\% (Method A) and 45\% (Method B). Anal. Calcd for $\mathrm{C}_{20} \mathrm{H}_{26} \mathrm{Cl}_{2} \mathrm{CuN}_{8} \mathrm{O}_{13}$ : C, 33.29; H, 3.60; N, 15.53. Found: C, 33.18; $\mathrm{H}, 3.76$; N, 15.45. IR (KBr; $\left.\mathrm{cm}^{-1}\right): 3362,3220\left(\mathrm{vb}, v_{\mathrm{OH}}\right), 1558(\mathrm{w}$, $\mathrm{C}=\mathrm{NPz}), 1110\left(\mathrm{br}, v_{\mathrm{ClO}_{4}}^{-}\right), 1065-1030\left(\mathrm{~ms}, v_{\mathrm{N}-\mathrm{NPz}}\right), 370\left(v_{\mathrm{Cu}-\mathrm{O}\left(\mathrm{H}_{2} \mathrm{O}\right)}\right)$, $262\left(v_{\mathrm{Cu}-\mathrm{NPz} \text { ring }}\right)$. DRS $\left(\lambda_{\max }, \mathrm{nm}\right): 547$. UV-vis $\left(\mathrm{MeOH}, \lambda_{\max }, \mathrm{nm}\right.$ $\left.\left(\epsilon, \mathrm{M}^{-1} \mathrm{~cm}^{-1}\right)\right): 721$ (47.4) (broad). UV-vis (DMF, $\lambda_{\max }, \mathrm{nm}(\epsilon$, $\left.\mathrm{M}^{-1} \mathrm{~cm}^{-1}\right)$ ): 778 (47.4) (broad). UV-vis (DMSO, $\lambda_{\max }, \mathrm{nm}\left(\epsilon, \mathrm{M}^{-1}\right.$ $\left.\mathrm{cm}^{-1}\right)$ ): 854 (51) (broad). Molar conductivity ( $\mathrm{MeOH}$ solution): $\Lambda_{\mathrm{m}}=204 \Omega^{-1} \mathrm{~cm}^{2} \mathrm{~mol}^{-1}$. Magnetism (solid state, room temperature): $\mu_{\mathrm{eff}}=1.63 \mu_{\mathrm{B}}$.

$\left[\mathrm{Co}\left(\mathrm{HMPz}_{3}\right.\right.$ tren $\left.)\right] \mathrm{ClO}_{4}(\mathbf{2})$. A mixture of tren $(0.146 \mathrm{~g}, 1 \mathrm{mmol})$ and MPA (0.33 g, $3 \mathrm{mmol})$ in EtOH (30 mL) was refluxed for $2 \mathrm{~h}$. The resulting light yellow solution was cooled and filtered. To the filtrate was added $\mathrm{Co}\left(\mathrm{ClO}_{4}\right)_{2} \cdot 6 \mathrm{H}_{2} \mathrm{O}(0.37 \mathrm{~g}, 1 \mathrm{mmol})$, and the mixture refluxed again for $2 \mathrm{~h}$. The clear red solution obtained thereby was allowed to evaporate slowly. A red amorphous product was obtained after 7 days which was collected by filtration, washed with ethanol, and dried over fused $\mathrm{CaCl}_{2}$ (yield 60\%). The product was recrystallized from $\mathrm{CH}_{3} \mathrm{CN}$. X-ray quality crystals were grown by slow diffusion of benzene into $\mathrm{CH}_{3} \mathrm{CN}$ solution of the compound. Anal. Calcd for $\mathrm{C}_{21} \mathrm{H}_{28} \mathrm{ClCoN}_{10} \mathrm{O}_{4}$ : C, 43.53; H, 4.83; N, 24.18. Found: C, 43.41; H, 4.90; N, 24.03. IR (KBr; $\left.\mathrm{cm}^{-1}\right): 3419,3220$

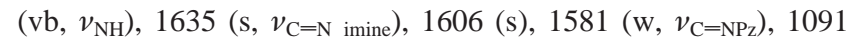
(vb), 624 (s, $\left.v_{\mathrm{ClO}_{4}}{ }^{-}\right)$. DRS $\left(\lambda_{\max }, \mathrm{nm}\right): 375,485$. UV-vis: no well-

(28) Barik, A. K.; Paul, S.; Kar, S. K.; Butcher, R. J.; Bryan, J. C. Polyhedron 1999, 18, 571. defined band in visible region. Molar conductivity (DMF solution): $\Lambda_{\mathrm{m}}=89 \Omega^{-1} \mathrm{~cm}^{2} \mathrm{~mol}^{-1} .{ }^{1} \mathrm{H} \mathrm{NMR}\left(d_{6}\right.$-DMSO, $\left.300 \mathrm{MHz}, 298 \mathrm{~K}\right)$ : $\delta 2.20(\mathrm{~s}, 9 \mathrm{H}, \mathrm{Me}), 2.99\left(\mathrm{~m}, 6 \mathrm{H}, \mathrm{N}-\mathrm{CH}_{2}\right), 3.17(\mathrm{~m}, 6 \mathrm{H},=\mathrm{N}-$ $\left.\mathrm{CH}_{2}\right), 6.64\left(\mathrm{~s}, 3 \mathrm{H}, \mathrm{C}_{4}-\mathrm{H}-\mathrm{Pz}\right), 8.26(\mathrm{~s}, 3 \mathrm{H},-\mathrm{N}=\mathrm{C}-\mathrm{H}), 14.2(\mathrm{~s}$, $1 \mathrm{H}, \mathrm{Pz}-\mathrm{NH})$.

$\left[\mathrm{Zn}\left(\mathrm{H}_{3} \mathrm{MPz}_{3}\right.\right.$ tren $\left.)\right]\left(\mathrm{ClO}_{4}\right)_{2}$ (3). The compound was prepared by following method $\mathrm{A}$, except that $\mathrm{Zn}\left(\mathrm{ClO}_{4}\right)_{2} \cdot 6 \mathrm{H}_{2} \mathrm{O}$ was used as the metal salt. Upon slow evaporation at room temperature, a sticky mass was obtained. It was dissolved in $\mathrm{CH}_{3} \mathrm{CN}$ and purified on a silica gel column using 1:1 (v/v) benzene/acetonitrile mixture as eluant. The yellow fraction obtained was evaporated and solidified by scratching after addition of dry ether. Unfortunately, we were unable to grow $\mathrm{X}$-ray quality crystals of this yellow microcrystalline compound. Anal. Calcd for $\mathrm{C}_{21} \mathrm{H}_{30} \mathrm{Cl}_{2} \mathrm{~N}_{10} \mathrm{ZnO}_{8}$ : C, 36.7; H, 4.37; $\mathrm{N}, 20.39$. Found: C, 37.08; H, 4.12; N, 20.55. ${ }^{1} \mathrm{H}$ NMR $\left(d_{6}\right.$-DMSO, $300 \mathrm{MHz}, 298 \mathrm{~K}): \delta 2.27(\mathrm{~s}, 9 \mathrm{H}, \mathrm{Me}), 2.85\left(\mathrm{~m}, 6 \mathrm{H}, \mathrm{N}-\mathrm{CH}_{2}\right.$ ), $3.13\left(\mathrm{~m}, 6 \mathrm{H},=\mathrm{N}-\mathrm{CH}_{2}\right), 6.61\left(\mathrm{~s}, 3 \mathrm{H}, \mathrm{C}_{4}-\mathrm{H}-\mathrm{Pz}\right), 8.55(\mathrm{~s}, 3 \mathrm{H}$, $\mathrm{N}=\mathrm{C}-H), 13.0(\mathrm{~s}, 3 \mathrm{H}, \mathrm{Pz}-\mathrm{NH})$.

Physical Methods. UV - vis spectra were measured on a Hitachi U-3501 spectrophotometer. Infrared spectra were recorded on $\mathrm{KBr}$ disks (4000-200 $\mathrm{cm}^{-1}$ ) with a JASCO FTIR model 420 spectrophotometer. ${ }^{1} \mathrm{H}$ NMR spectra were recorded with a Bruker AM300L (300 MHz) superconducting FT NMR. EPR spectra were obtained using a Varian E-112 spectrometer operating at X-band. The field was calibrated with a powder sample of DPPH $(g=2.0037)$. Solvents used were $\mathrm{MeOH} / \mathrm{EtOH}(1: 4 \mathrm{v} / \mathrm{v})$ with copper complex concentration approximately $10^{-3} \mathrm{M}$. Room temperature magnetic moments were measured with a PAR 155 vibrating sample magnetometer. The diffuse reflectance spectra of the reported complexes were recorded on a Hitachi U-3501 spectrophotometer. Electrical conductivity measurements were carried out in $\mathrm{MeOH}$ or DMF solution using a Systronics model 304 digital conductivity meter. Elemental analyses were carried out at IACS, Calcutta, with a Perkin-Elmer model $2400 \mathrm{CHN}$ analyzer. Cyclic voltammetry was carried out using Sycopel model AEW2 1820F/S instrument. The measurements were performed at $300 \mathrm{~K}$ in DMF solutions containing $0.2 \mathrm{M} \mathrm{TBAP}^{24}$ and $10^{-3}-10^{-4} \mathrm{M} \mathrm{Cu}(\mathrm{II})$ complex deoxygenated by bubbling with nitrogen. The working, counter, and reference electrodes used were a platinum wire, a platinum coil, and an SCE. Thermogravimetric analysis was carried out using Mettler Toledo model TGA/SDTA $851^{\mathrm{e}}$ instrument.

X-ray Crystal Structure Analysis. Relevant crystallographic data are collected in Table 1 . Intensity data for the compounds were measured on an Enraf-Nonius CAD 4 diffractometer using graphitemonochromated Mo K $\alpha$ radiation $(\lambda=0.71073 \AA)$ in the $\omega-2 \theta$ scan mode. These were corrected for Lorentz-polarization effects. The structures were solved by using the SHELXS-97 package of programs and refined by full-matrix least-squares technique based on $F^{2}$ (SHELXL-97). Hydrogen atoms were added in the calculated positions.

\section{Results and Discussion}

The coordination compound of the expected tripodal ligand was synthesized in situ by template methodology in the presence of $\mathrm{Co}(\mathrm{II})$ and $\mathrm{Zn}(\mathrm{II})$ ions.

The ligand $\left(\mathrm{H}_{3} \mathrm{MPz}_{3}\right.$ tren $)$ shows different extents of deprotonation in these complexes. In the cobalt(III) compound [Co( $\mathrm{HMPz}_{3}$ tren) $\mathrm{ClO}_{4}(\mathbf{2})$, it loses two protons, while, in the corresponding $\mathrm{Zn}(\mathrm{II})$ complex $\left[\mathrm{Zn}\left(\mathrm{H}_{3} \mathrm{MPz}_{3}\right.\right.$ tren $\left.)\right]\left(\mathrm{ClO}_{4}\right)_{2}$ (3), it remains completely neutral. The same reaction when carried out with $\mathrm{Cu}$ (II) ion followed an unprecedented path, leading to porphyrinogen complexes $(\mathbf{1 a}, \mathbf{1 b})$ involving the 
Paul et al.

Table 1. Crystal Data and Structure Refinement

\begin{tabular}{|c|c|c|c|}
\hline & $\mathrm{C}_{20} \mathrm{H}_{26} \mathrm{CuN}_{10} \mathrm{O}_{11}$ & $\mathrm{C}_{20} \mathrm{H}_{26} \mathrm{Cl}_{2} \mathrm{CuN}_{8} \mathrm{O}_{13}$ & $\mathrm{C}_{21} \mathrm{H}_{28} \mathrm{ClCoN}_{10} \mathrm{O}_{4}$ \\
\hline fw & 646.05 & 720.93 & 578.91 \\
\hline$T$ & 293(2) K & 295(2) K & 295(2) K \\
\hline wavelength & $0.71073 \AA$ & $0.71073 \AA$ & $0.71073 \AA$ \\
\hline cryst syst & monoclinic & monoclinic & monoclinic \\
\hline space group & $C 2 / c$ & $C 2 / c$ & $C 2 / c$ \\
\hline \multirow{6}{*}{$\begin{array}{l}\text { unit cell } \\
\quad \text { dimensions }\end{array}$} & $a=19.476(3) \AA$ & $a=20.967(3) \AA$ & $a=21.293(3) \AA$ \\
\hline & $b=9.4116(8) \AA$ & $b=9.1563(18) \AA$ & $b=12.724(2) \AA$ \\
\hline & $c=14.204(3) \AA$ & $c=14.858(4) \AA$ & $c=19.777(4) \AA$ \\
\hline & $\alpha=90^{\circ}$ & $\alpha=90^{\circ}$ & $\alpha=90^{\circ}$ \\
\hline & $\beta=107.58(2)^{\circ}$ & $\beta=108.44(3)^{\circ}$ & $\beta=93.03(2)^{\circ}$ \\
\hline & $\gamma=90^{\circ}$ & $\gamma=90^{\circ}$ & $\gamma=90^{\circ}$ \\
\hline$V, Z$ & $2482.0(7) \AA^{3}, 4$ & $2706.0(10) \AA^{3}, 4$ & $5350.6(15) \AA^{3}, 8$ \\
\hline$D($ calcd $)$ & $1.729 \mathrm{mg} / \mathrm{m}^{3}$ & $1.770 \mathrm{mg} / \mathrm{m}^{3}$ & $1.437 \mathrm{mg} / \mathrm{m}^{3}$ \\
\hline abs coeff & $0.964 \mathrm{~mm}^{-1}$ & $1.088 \mathrm{~mm}^{-1}$ & $0.788 \mathrm{~mm}^{-1}$ \\
\hline$F(000)$ & 1332 & 1476 & 2400 \\
\hline cryst size & $\begin{array}{c}0.60 \times 0.20 \times \\
0.05 \mathrm{~mm}^{3}\end{array}$ & $\begin{array}{c}0.40 \times 0.20 \times \\
0.10 \mathrm{~mm}^{3}\end{array}$ & $\begin{array}{c}0.40 \times 0.25 \times \\
0.20 \mathrm{~mm}^{3}\end{array}$ \\
\hline $\begin{array}{l}\theta \text { range for data } \\
\text { collection }\end{array}$ & $2.19-25.00^{\circ}$ & $2.05-25.00^{\circ}$ & $1.87-25.00^{\circ}$ \\
\hline limiting indices & $\begin{array}{l}-23 \leq h \leq 22 \\
0 \leq k \leq 11 \\
0 \leq l \leq 16\end{array}$ & $\begin{array}{l}-24 \leq h \leq 23 \\
0 \leq k \leq 10 \\
0 \leq l \leq 17\end{array}$ & $\begin{array}{l}-25 \leq h \leq 25 \\
0 \leq k \leq 15 \\
0 \leq l \leq 23\end{array}$ \\
\hline reflns collected & 2194 & 2385 & 4718 \\
\hline indep reflns & $\begin{array}{c}2194\left(R_{\text {int }}=\right. \\
0.0000)\end{array}$ & $\begin{array}{c}2385\left(R_{\text {int }}=\right. \\
0.0000)\end{array}$ & $\begin{array}{c}4716\left(R_{\text {int }}=\right. \\
0.0000)\end{array}$ \\
\hline $\begin{array}{c}\text { completeness to } \\
\theta=25.00^{\circ}\end{array}$ & $100 \%$ & $100 \%$ & $100 \%$ \\
\hline abs correction & $\begin{array}{c}\text { empirical used } \\
\psi \text {-scans }\end{array}$ & $\begin{array}{l}\text { empirical used } \\
\psi \text {-scans }\end{array}$ & $\begin{array}{l}\text { empirical used } \\
\psi \text {-scans }\end{array}$ \\
\hline $\begin{array}{l}\underset{\text { max and } \min }{\text { transm }} \\
\text {. }\end{array}$ & $\begin{array}{c}0.6731 \text { and } \\
0.6252\end{array}$ & $\begin{array}{c}0.8187 \text { and } \\
0.7322\end{array}$ & $\begin{array}{c}0.8540 \text { and } \\
0.7914\end{array}$ \\
\hline $\begin{array}{l}\text { refinement } \\
\text { method }\end{array}$ & $\begin{array}{l}\text { full-matrix least- } \\
\text { squares on } F^{2}\end{array}$ & $\begin{array}{l}\text { full-matrix least- } \\
\text { squares on } F^{2}\end{array}$ & $\begin{array}{l}\text { full-matrix least- } \\
\quad \text { squares on } F^{2}\end{array}$ \\
\hline $\begin{array}{l}\text { data/restraints/ } \\
\text { params }\end{array}$ & $2194 / 0 / 197$ & $2385 / 0 / 201$ & $4718 / 0 / 334$ \\
\hline GOF on $F^{2}$ & 1.034 & 1.045 & 1.017 \\
\hline \multirow[t]{2}{*}{$\begin{array}{c}\text { final } R \text { indices } \\
\quad[I>2 \sigma(I)]\end{array}$} & $\mathrm{R} 1=0.0414$ & $\mathrm{R} 1=0.0495$ & $\mathrm{R} 1=0.0545$ \\
\hline & $\mathrm{wR} 2=0.1119$ & $w \mathrm{R} 2=0.1424$ & $w R 2=0.1892$ \\
\hline \multirow[t]{2}{*}{$\begin{array}{l}\mathrm{R} \text { indices } \\
\quad \text { (all data) }\end{array}$} & $\mathrm{R} 1=0.0656$ & $\mathrm{R} 1=0.0830$ & $\mathrm{R} 1=0.1033$ \\
\hline & $w R 2=0.1227$ & $\mathrm{wR} 2=0.1601$ & $\mathrm{wR} 2=0.2142$ \\
\hline $\begin{array}{l}\text { largest diff peak } \\
\text { and hole }\end{array}$ & $\begin{array}{l}0.773 \text { and } \\
\quad-0.352 \mathrm{e}^{-3}\end{array}$ & $\begin{array}{l}0.659 \text { and } \\
\quad-0.403 \text { e } \AA^{-3}\end{array}$ & $\begin{array}{l}1.075 \text { and } \\
\quad-0.426 \mathrm{e} \AA^{-3}\end{array}$ \\
\hline
\end{tabular}

pyrazole moiety as the building blocks. Despite our repeated attempts, we were unable to isolate the porphyrinogen molecule in the free state. When $\mathrm{Cu}$ (II) ion is removed from the porphyrinogen complexes (1a and $\mathbf{1 b}$ ) by sulfide precipitation, the product obtained is a pale yellow microcrystalline solid with a ${ }^{1} \mathrm{H}$ NMR spectrum $\left[{ }^{1} \mathrm{H}\right.$ NMR $\left(d_{6^{-}}\right.$ DMSO, $300 \mathrm{MHz}, 298 \mathrm{~K}): \delta 2.18$ (s, 3H, Me), 6.51 (s, $\mathrm{C}_{4}-$ $\mathrm{H}-\mathrm{Pz}), 9.55(\mathrm{~s}, 1 \mathrm{H}, \mathrm{CHO})]$ typical of the precursor aldehyde, ${ }^{28}$ viz. MPA. The macrocycle formation fails to occur in the presence of other metal ions. The study of the TGA curve of complex 1a confirms the loss of the coordinated water molecule in the temperature range 148$225{ }^{\circ} \mathrm{C}$. The tripodal acts as $\mathrm{N}_{6}$-binegative for $\mathrm{Co}(\mathrm{III})$ complex because of the deprotonation of two $\mathrm{N}-\mathrm{H}(\mathrm{Pz})$. The coordination polyhedron of the said complex is best described as distorted octahedral. The $\mathrm{Co}-\mathrm{N}(10)$ distance $(3.289 \AA)$ shows that there is no bonding. The crystal structures of the $\mathrm{Cu}$ (II) complexes and the $\mathrm{Co}(\mathrm{III})$ complex are described in the following paragraphs.

Description of Crystal Structures: 1a and $1 \mathbf{b}$. The ORTEP plot of the cationic portion of $\mathbf{1 a}$ is represented in
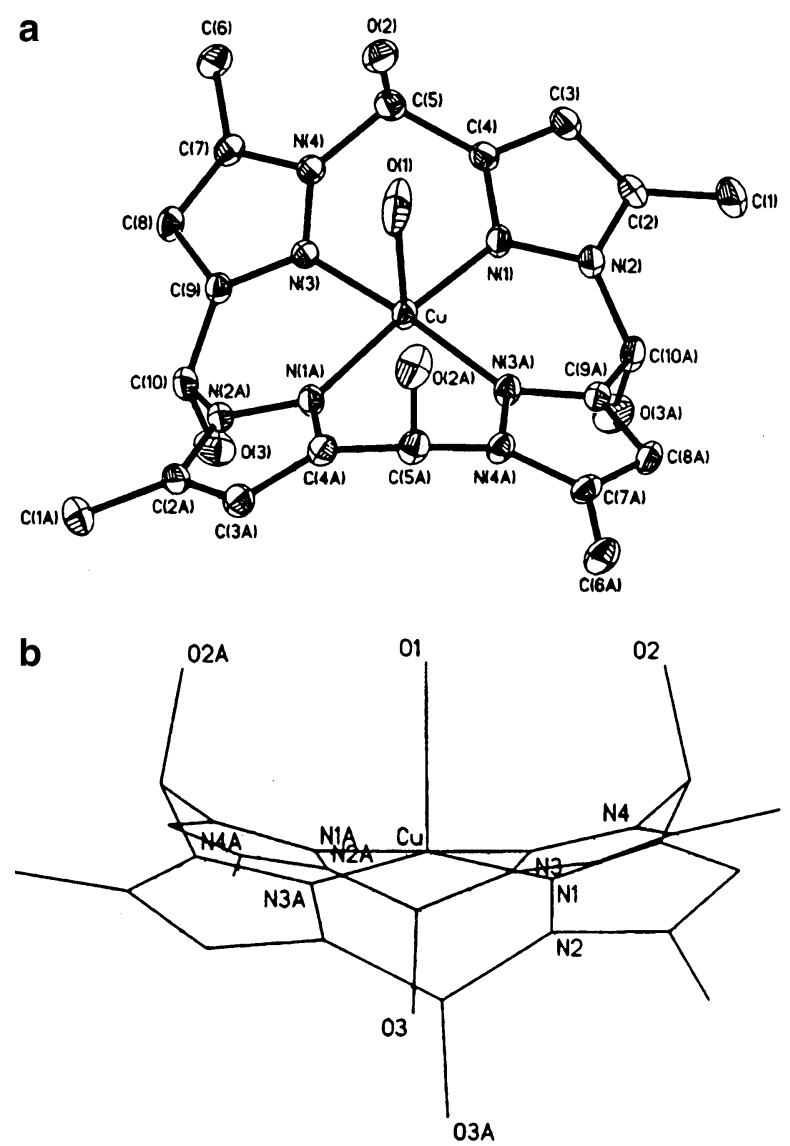

Figure 2. (a) ORTEP plot of the cation of 1a. The thermal ellipsoids are drawn at the $30 \%$ probability level, and the hydrogen atoms are omitted for clarity. (b) Saddle distortion form of 1a.

Table 2. Bond lengths $(\AA)$ and Angles (deg) for $\mathbf{1 a}$ and $\mathbf{1 b}$

\begin{tabular}{llll}
\hline \multicolumn{5}{c}{$1 \mathbf{a}$} & \multicolumn{3}{c}{$\mathbf{1 b}$} \\
\hline \multicolumn{5}{c}{ Bond Lengths } \\
$\mathrm{Cu}-\mathrm{N}(1) \# 1$ & $1.955(3)$ & $\mathrm{Cu}-\mathrm{N}(1) \# 1$ & $1.941(4)$ \\
$\mathrm{Cu}-\mathrm{N}(3) \# 1$ & $1.955(2)$ & $\mathrm{Cu}-\mathrm{N}(3) \# 1$ & $1.949(4)$ \\
$\mathrm{Cu}-\mathrm{O}(1)$ & $2.240(5)$ & $\mathrm{Cu}-\mathrm{O}(1)$ & $2.338(5)$ \\
\multicolumn{5}{c}{ Bond Angles } \\
$\mathrm{N}(1) \# 1-\mathrm{Cu}-\mathrm{N}(1)$ & $171.40(18)$ & $\mathrm{N}(1) \# 1-\mathrm{Cu}-\mathrm{N}(1)$ & $173.4(2)$ \\
$\mathrm{N}(1)-\mathrm{Cu}-\mathrm{N}(3)$ & $89.33(22)$ & $\mathrm{N}(1)-\mathrm{Cu}-\mathrm{N}(3) \# 1$ & $90.43(17)$ \\
$\mathrm{N}(1)-\mathrm{Cu}-\mathrm{N}(3) \# 1$ & $89.89(12)$ & $\mathrm{N}(1)-\mathrm{Cu}-\mathrm{N}(3)$ \\
$\mathrm{N}(1) \# 1-\mathrm{Cu}-\mathrm{N}(3)$ & $89.89(12)$ & $\mathrm{N}(1) \# 1-\mathrm{Cu}-\mathrm{N}(3) \# 1$ & $89.09(16)$ \\
$\mathrm{N}(1) \#-\mathrm{Cu}-\mathrm{N}(3) \# 1$ & $89.33(12)$ & $\mathrm{N}(1) \# 1-\mathrm{Cu}-\mathrm{N}(3)$ & $90.43(17)$ \\
$\mathrm{N}(3)-\mathrm{Cu}-\mathrm{N}(3) \# 1$ & $169.61(18)$ & $\mathrm{N}(3) \# 1-\mathrm{Cu}-\mathrm{N}(3)$ & $171.6(2)$ \\
$\mathrm{N}(1) \# 1-\mathrm{Cu}-\mathrm{O}(1)$ & $94.30(9)$ & $\mathrm{N}(1) \# 1-\mathrm{Cu}-\mathrm{O}(1)$ & $93.28(11)$ \\
$\mathrm{N}(3)-\mathrm{Cu}-\mathrm{O}(1)$ & $95.20(9)$ & $\mathrm{N}(3) \# 1-\mathrm{Cu}-\mathrm{O}(1)$ & $94.19(11)$ \\
$\mathrm{N}(1)-\mathrm{Cu}-\mathrm{O}(1)$ & $94.30(9)$ & $\mathrm{N}(1)-\mathrm{Cu}-\mathrm{O}(1)$ & $93.28(11)$ \\
$\mathrm{N}(3) \# 1-\mathrm{Cu}-\mathrm{O}(1)$ & $95.20(9)$ & $\mathrm{N}(3)-\mathrm{Cu}-\mathrm{O}(1)$ & $94.19(11)$
\end{tabular}

Figure 2a. Selected bond angles and distances of $\mathbf{1 a}$ and $\mathbf{1 b}$ are displayed in Table 2 . The coordination geometry of the copper(II) atom in both the complexes reveals a distorted square pyramidal geometry. The geometry is completed by the oxygen atom of the water molecule in the apical site and four nitrogen donors from the unusual macrocyclic ligand occupying the basal plane. The $\mathrm{Cu}(\mathrm{II})$ atom is shifted approximately by $0.162 \AA$ out of the basal plane for $1 \mathbf{a}(0.126$ $\AA$ for $1 \mathbf{b})$ toward the apical $\mathrm{O}$ atom. All the $\mathrm{Cu}-\mathrm{N}$ bond distances are same in $\mathbf{1 a}(1.955 \AA)$ and almost same in $\mathbf{1 b}$ (1.941 and $1.949 \AA$ ). These distances are shorter than similar bonds reported in other macrocyclic compounds ${ }^{26,29}$ and comparable with those reported for $\left[\mathrm{Cu}_{2}\left(\mathrm{~N}_{6} \mathrm{O}\right)(\mathrm{OH})\right]\left(\mathrm{BF}_{4}\right)_{2}$, 
${ }^{30}[\mathrm{Cu}(\mathrm{II})$ (pmea) $\mathrm{Cl}] \mathrm{ClO}_{4} \cdot \mathrm{H}_{2} \mathrm{O},{ }^{31}$ and $[\mathrm{Cu}($ appn $)]\left(\mathrm{ClO}_{4}\right)_{2} \cdot \mathrm{H}_{2} \mathrm{O}$ $\left(\mathrm{Cu}_{2} \mathrm{Zn}_{2} \mathrm{SOD}\right.$ model $){ }^{32}$ The distortion induced in the presence of the pyrazole ring is reflected in the $\mathrm{N}(1) \# 1-\mathrm{Cu}-\mathrm{N}(1)$ and $\mathrm{N}(3)-\mathrm{Cu}-\mathrm{N}(3) \# 1$ bond angles. These are $171.40(18)^{\circ}$ and $169.61(18)^{\circ}$ for $\mathbf{1 a}\left(173.4(2)^{\circ}\right.$ and $171.6(2)^{\circ}$ for $\left.\mathbf{1 b}\right)$, respectively.

The mean deviation of the basal plane N1A, N3, N1, and $\mathrm{N} 3 \mathrm{~A}$ is only $0.0151 \AA$ ( $0.0156 \AA$ for $\mathbf{1 b})$. The oxygen atom of the water molecule completes the coordination sphere by forming a bond to the metal at a distance of 2.240(5) $\AA$ [2.338(15) $\AA$ for $\mathbf{1 b}]$. In case of the perchlorate complex (1b), the $\mathrm{Cu}-\mathrm{O}$ distance is within the range expected for an axially bound aquo molecule. ${ }^{32,33}$ This value is somewhat shorter in the case of the nitrate complex (1a), and this shorter value may be compared with a bond length of $2.263 \AA$ obtained for the complex $\left[\mathrm{CuL}^{8}\right]\left[\mathrm{H}_{2} \mathrm{~L}^{8}=1,4,8,11\right.$-tetraazacyclotetradecane-1,8-diacetic acid]. ${ }^{34}$ If the angle $\mathrm{N}(1)-\mathrm{Cu}-$ $\mathrm{O}(1)$ is less than $80^{\circ}$, the coordinated $\mathrm{O}$-atoms are laterally displaced from axial coordination sites. ${ }^{29}$ In 1a, the average angle value of $94.75(9)^{\circ}\left[93.74(11)^{\circ}\right.$ for $\mathbf{1 b}$ ] strongly supports the axial coordination of the $\mathrm{O}$-atom. The $\mathrm{H}_{2} \mathrm{O}$ molecule of the axial coordination site is also H-bonded in both the complexes with their corresponding anions.

As the H-bond distance for the nitrate complex $(2.665 \AA)$ is shorter than that of the perchlorate complex $(2.860 \AA)$, a strong hydrogen bonding is indicated for the former and a moderate one for the latter. ${ }^{32,35}$

Crystal structure studies show that the four $\mathrm{OH}$ groups present in the four chiral carbon atoms of $\mathrm{T}_{3}$-porphyrinogen are in a trans disposition above and below the porphyrinogen mean plane. Interestingly, the pyrazole rings are situated downward with respect to the $\mathrm{Cu}$ atom. These observations reveal that the porphyrinogen ring is in a saddle-distorted form (Figure 2b).

Compound 2. An ORTEP plot of the cationic portion of 2 is presented in Figure 3. Selected metrical parameters are listed in Table 3. The potentially heptadentate $\mathrm{H}_{3} \mathrm{MPz}_{3}$ tren ligand coordinates to the $\mathrm{Co}(\mathrm{III})$ ion through the three imine and three pyrazole nitrogen atoms.

It is noteworthy that the bond angle $\mathrm{C}-\mathrm{N}-\mathrm{C}$ at $\mathrm{N}(10)$ is ca. $119^{\circ}$ which is also in agreement with our previously determined value for the complex $\left[\mathrm{Ni}_{(}\left(\mathrm{H}_{3} \mathrm{MPz}_{3}\right.\right.$ tren $\left.)\right]\left(\mathrm{BF}_{4}\right)_{2}$. $0.5 \mathrm{H}_{2} \mathrm{O} .{ }^{27}$ The tertiary amine nitrogen atom $\mathrm{N}(10)$ appears to be the apex of a three-ribbed umbrella that has been inverted by the winds. Among different parameters, the height-to-bite ratio, $h / b$, is useful for describing the coordination polyhedra. An $h / b$ ratio of 0.728 supports the octahedral geometry of the complex. ${ }^{17}$

(29) Bernhardt, P. V.; Sharpe, P. C. Inorg. Chem. 2000, 39, 2020-2025

(30) Sorrell, T. N.; Jameson, D. L.; O’Connor, C. J. Inorg. Chem. 1984, 23, 190.

(31) Schatz, M.; Becker, M.; Thaler, F.; Hampel, F.; Schindler, S.; Jacobson, R. R.; Tyekler, Z.; Murthy, N. N.; Ghosh, P.; Chen, Q.; Zubieta, J.; Karlin, K. D. Inorg. Chem. 2001, 41, 2312-2322.

(32) Liu, C.-M.; Xiong, R.-G.; You, X.-Z. Polyhedron 1997, 16, 119.

(33) Orpen, A. G.; Brammer, L.; Allen, F. H.; Kennard, O.; Watson, D. G.; Taylor, R. J. Chem. Soc., Dalton Trans. 1989, 1.

(34) Chapman, J.; Ferguson, G.; Gallaghar, J. F.; Jennings, M. C.; Parker, D. J. Chem. Soc., Dalton Trans. 1992, 345.

(35) Cotton, F. A.; Wilkinson, G. Advanced Inorganic Chemistry, 5th ed.; Wiley: New York, 1988; p 93.
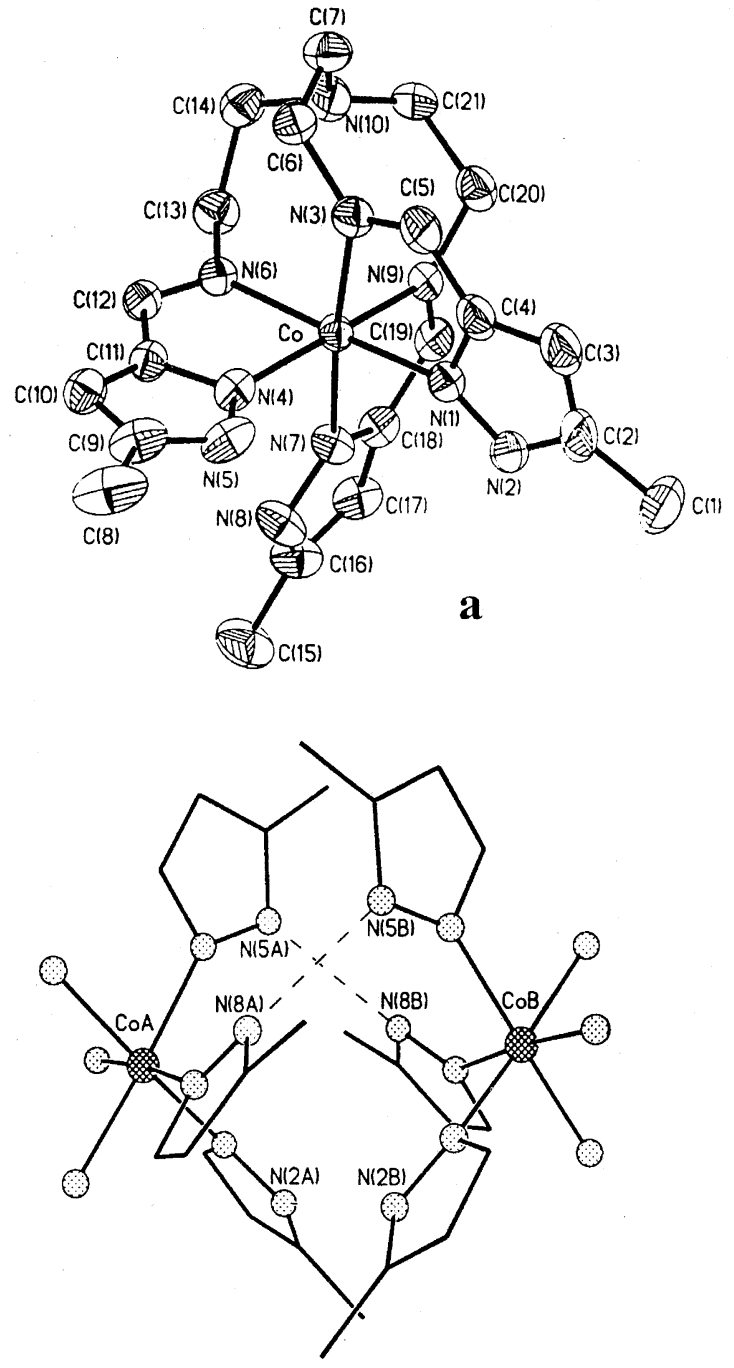

b

Figure 3. (a) ORTEP plot of the cation of 2. The thermal ellipsoids are drawn at the $30 \%$ probability level, and the hydrogen atoms are omitted for clarity. (b) Hydrogen bonding mode of 2.

Table 3. Selected Metrical Parameters for 2

\begin{tabular}{lrlr}
\multicolumn{2}{c}{ Bond Lengths } & \multicolumn{2}{c}{ Bond Angles } \\
$\mathrm{Co}-\mathrm{N}(4)$ & $2.014(5)$ & $\mathrm{N}(4)-\mathrm{Co}-\mathrm{N}(7)$ & $95.13(17)$ \\
$\mathrm{Co}-\mathrm{N}(1)$ & $2.016(5)$ & $\mathrm{N}(7)-\mathrm{Co}-\mathrm{N}(1)$ & $97.08(19)$ \\
$\mathrm{Co}-\mathrm{N}(7)$ & $2.015(4)$ & $\mathrm{N}(7)-\mathrm{Co}-\mathrm{N}(9)$ & $78.78(17)$ \\
$\mathrm{Co}-\mathrm{N}(3)$ & $2.055(5)$ & $\mathrm{N}(4)-\mathrm{Co}-\mathrm{N}(3)$ & $88.86(17)$ \\
$\mathrm{Co}-\mathrm{N}(9)$ & $2.052(4)$ & $\mathrm{N}(1)-\mathrm{Co}-\mathrm{N}(3)$ & $79.01(19)$ \\
$\mathrm{Co}-\mathrm{N}(6)$ & $2.055(4)$ & $\mathrm{N}(4)-\mathrm{Co}-\mathrm{N}(6)$ & $78.75(17)$ \\
$\mathrm{N}(1)-\mathrm{N}(2)$ & $1.314(7)$ & $\mathrm{N}(1)-\mathrm{Co}-\mathrm{N}(6)$ & $173.58(19)$ \\
$\mathrm{N}(4)-\mathrm{N}(5)$ & $1.350(6)$ & $\mathrm{N}(3)-\mathrm{Co}-\mathrm{N}(6)$ & $95.83(17)$ \\
$\mathrm{N}(7)-\mathrm{N}(8)$ & $1.343(6)$ & $\mathrm{N}(4)-\mathrm{Co}-\mathrm{N}(1)$ & $97.22(18)$ \\
& & $\mathrm{N}(4)-\mathrm{Co}-\mathrm{N}(9)$ & $171.97(17)$ \\
& & $\mathrm{N}(1)-\mathrm{Co}-\mathrm{N}(9)$ & $88.79(18)$ \\
& & $\mathrm{N}(7)-\mathrm{Co}-\mathrm{N}(3)$ & $174.75(18)$ \\
& & $\mathrm{N}(9)-\mathrm{Co}-\mathrm{N}(3)$ & $97.54(18)$ \\
& & $\mathrm{N}(7)-\mathrm{Co}-\mathrm{N}(6)$ & $88.30(17)$ \\
& & $\mathrm{N}(9)-\mathrm{Co}-\mathrm{N}(6)$ & $95.71(17)$
\end{tabular}

From the $\mathrm{Co}-\mathrm{N}$ bond distance values (Table 3 ), it is observed that the three $\mathrm{Co}-\mathrm{N}(\mathrm{Pz})$ bond distances (average distance $2.015 \AA$ ) are shorter than $\mathrm{Co}-\mathrm{N}$ (iminyl) bond distances (average distance $2.054 \AA$ ). This shortening of bond distance suggests that the $\mathrm{N}$ (pyrazolyl) atoms are the negative donor sites. 
Paul et al.

From the crystal packing diagram (Figure 3b), it is evident that the two Co units are linked together through H-bonding. As there is only one $\mathrm{N}-\mathrm{H}$ (pyrazolyl) among the three uncoordinated N(pyrazolyl) (other two are deprotonated and satisfy the primary valency of $\mathrm{Co}(\mathrm{III})$ ion), there are two $\mathrm{H}$-bonding possibilities. Figure $3 \mathrm{~b}$ shows both the $\mathrm{H}$ bondings, $\operatorname{CoAN}(5 \mathrm{~A}) \cdots \mathrm{N}(8 \mathrm{~B}) \mathrm{CoB}$ and $\operatorname{CoAN}(8 \mathrm{~A}) \cdots \mathrm{N}(5 \mathrm{~B})-$ CoB. Both the distances are of same value $(2.777 \AA)$ indicating moderately strong H-bonding. ${ }^{32,35}$

Diffuse Reflectance and Solution Spectra. The diffuse reflectance spectra of the $\mathrm{Cu}$-complexes exhibit only one broad band around $566 \mathrm{~nm}$ for $\mathbf{1 a}$ and $547 \mathrm{~nm}$ for $\mathbf{1 b}$ indicating the square pyramidal geometry of the complex species. Similar observations with a little deviation in band position are also reported for the same chromophore $\mathrm{CuN}_{4} \mathrm{O}$ where $\mathrm{N}$-donor sites are from pyrazole rings $\mathrm{s}^{13,36,37}$ and also for single-crystal spectra of chelate complex $\left[\mathrm{Cu}(1,3-\mathrm{Pn})_{2}\right.$. $\left.\mathrm{H}_{2} \mathrm{O}\right] \mathrm{SO}_{4}{ }^{38}$ Probably, the macrocyclic ring effect may be responsible for creating such deviation. In $\mathrm{MeOH}$ solution, the complexes display absorption bands at $718 \mathrm{~nm}(\epsilon=69.9$ $\left.\mathrm{M}^{-1} \mathrm{~cm}^{-1}\right)$ and $721 \mathrm{~nm}\left(\epsilon=47.4 \mathrm{M}^{-1} \mathrm{~cm}^{-1}\right)$ for $\mathbf{1 a}$ and $\mathbf{1 b}$, respectively. This corresponds to $\mathrm{d}-\mathrm{d}$ transition of $\mathrm{Cu}$ (II) in a weak tetragonal field and is in accordance with those reported for SOD mimicking compounds..$^{32,39-41}$ This shift in $\lambda_{\max }$ value in solution from that of the solid state may be due to replacement of the weakly coordinated $\mathrm{H}_{2} \mathrm{O}$ molecule in the fifth position by the solvent molecule maintaining the same geometry. ${ }^{37}$ This proposition is further substantiated from the EPR spectrum of the complex (discussed later). Interestingly, when the spectra are recorded in DMF solution, the $\lambda_{\max }$ is shifted toward higher wavelengths. This trend is also followed in DMSO solvent and to a much greater extent. The red shift in optical spectra may be attributed to the strong coordinating property of the solvent, which results in the lowering in energy gap between two spin states. Nonplanarity is known to cause a red shift in the optical spectra of porphyrins due to greater destabilization of the HOMO compared with the LUMO. ${ }^{42}$ In strongly coordinating solvents, the original nonplanarity of the $\mathrm{CuN}_{4}$ chromophore is further enhanced because of the formation of a stronger bond with the solvents at the apical position.

The diffuse reflectance spectrum of the Co-complex exhibiting two bands at $375 \mathrm{~nm}\left({ }^{1} \mathrm{~A}_{1 \mathrm{~g}} \rightarrow{ }^{1} \mathrm{~T}_{1 \mathrm{~g}}\right)$ and at 485 $\mathrm{nm}\left({ }^{1} \mathrm{~A}_{1 \mathrm{~g}} \rightarrow{ }^{1} \mathrm{~T}_{2 \mathrm{~g}}\right)$ indicates the low spin octahedral geometry of the $\left[\mathrm{Co}\left(\mathrm{HMPz}_{3} \text { tren }\right)\right]^{+}$cation. Probably, the other bands are masked by the intense charge-transfer bands in UV region. That in DMF solution the complex exhibits no band in the visible region may be due to intrusion of tails of intense

(36) Lever, A. B. P. Inorganic Electronic Spectroscopy; Elsevier: New York, 1984; pp 560-570.

(37) Deters, R.; Krämer, R. Inorg. Chem. Acta 1998, 269, 117-124.

(38) Hathaway, B. J.; Billing, D. E. Coord. Chem. Rev. 1970, 5, 143207.

(39) Long, L.-S.; Tong, Y.-X.; Yang, S.-P.; Chen, X.-M.; Ji, L.-N. Transition Met. Chem. (London) 1999, 24, 440-444.

(40) Lu, Q.; Shin, C.-Y.; Luo, Q.-H. Polyhedron 1993, 12, 2005.

(41) Luo, Q.; Lu, Q.; Dai, A.; Huang, L. J. Inorg. Biochem. 1993, 51, 655.

(42) Shelnutt, J. A.; Song, X.-Z.; Ma, J.-G.; Jia, S.-L.; Jentzen, W.; Medforth, C. Chem. Soc. Rev. 1998, 27, 31 and references therein.

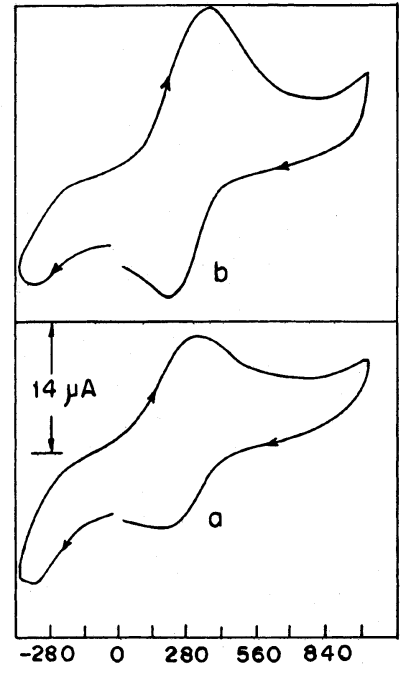

Figure 4. Cyclic voltammograms of 1a: (a) $100 \mathrm{mV} \mathrm{s}^{-1}$, (b) $250 \mathrm{mV}$ $\mathrm{s}^{-1}$. All potentials are given versus SCE. All measurements have been carried out in $0.2 \mathrm{M}$ TBAP/DMF at $300 \mathrm{~K}$.

charge-transfer bands into the visible portion of the spectrum, which masks the expected $\mathrm{d}-\mathrm{d}$ bands. ${ }^{43}$

EPR Spectroscopy. It is known that the EPR spectral pattern for square pyramidal complexes of $\mathrm{Cu}$ (II) $\left(\mathrm{d}_{x^{2}-y^{2}}\right.$ ground state (gs)) is quite different from that observed for $\mathrm{TBP}^{24}\left(\mathrm{~d}_{z^{2}} \mathrm{gs}\right)$. In general, frozen solution EPR spectra of square pyramidal complexes are characterized by an axial pattern with the features $g_{\|}>2.1>g_{\perp}>2.0$ and $\left|A_{\|}\right|=$ $120-150 \times 10^{-4} \mathrm{~cm}^{-1,44-48}$ whereas complexes with the TBP structure typically show EPR spectra having a "reversed axial" appearance with $g_{\perp}>g_{\|} \approx 2.0$ and $\left|A_{\|}\right|=(60-100)$ $\times 10^{-4} \mathrm{~cm}^{-1} \cdot{ }^{44,46-48}$ Thus, the coordination geometry around the central metal ion in the $\mathrm{Cu}$-complex can be deduced from an examination of the EPR spectral features. The EPR spectrum of 1a has been recorded in frozen solution $(\mathrm{MeOH} /$ $\mathrm{EtOH}, 1: 4 \mathrm{v} / \mathrm{v})$ at $77 \mathrm{~K}$ (X-band frequency). The spinHamiltonian parameters $g_{\|}(2.290)>2.1>g_{\perp}(2.067)>$ 2.0 and $A_{\|}=160 \times 10^{-4} \mathrm{~cm}^{-1}$ are typical of a square pyramidal geometry in solution. ${ }^{31}$ Here, the value $G>4.0$ indicates that the local tetragonal axes are aligned parallel or only slightly misaligned. ${ }^{38}$

Electrochemistry. The electron-transfer behavior of the $\mathrm{Cu}$ (II) complexes (1a and 1b) are grossly identical as revealed by cyclic voltammetry. A representative voltammogram for 1a in DMF is shown in Figure 4. It involves a quasireversible one-electron process at $E_{1 / 2}=0.260 \mathrm{~V}(0.262$ $\mathrm{V}$ for $\mathbf{1 b}$ ) versus SCE (scan rate $100 \mathrm{mV} / \mathrm{s}$ ). A comparison with the $\mathrm{Fe} / \mathrm{Fe}^{+}$couple indicates the involvement of a single electron in this redox process. ${ }^{49} \mathrm{We}$ believe this process to

(43) Barik, A. K.; Paul, S.; Butcher, R. J.; Kar, S. K. Polyhedron 2000, $19,2651$.

(44) Addison, A. W.; Hendriks, H. M. J.; Reedijk, J.; Thompson, L. K. Inorg. Chem. 1981, 20, 103-110.

(45) Duggan, M.; Ray, N.; Hathaway, B.; Tomlinson, G.; Briant, P.; Plein, K. J. Chem. Soc., Dalton Trans. 1980, 1342-1348.

(46) Nishida, Y.; Oishi, N.; Kida, S. Inorg. Chim. Acta 1980, 44, L257L258.

(47) Takahashi, K.; Ogawa, E.; Oishi, N.; Kida, S. Inorg. Chim. Acta 1982, 66, $97-103$.

(48) Morpurgo, L.; Falcioni, R.; Rotildo, G.; Desideri, A.; Mondovi, B. Inorg. Chim. Acta 1978, 28, L141-L143. 
be due either to a $\mathrm{Cu}(\mathrm{II}) / \mathrm{Cu}(\mathrm{III})$ electron transfer or a ligandbased process. Coulometric confirmation of electron stoichiometry was vitiated by the slow decomposition of these molecules in the time scale of this measurement.

\section{Conclusion and Comments}

Using the same synthetic procedure, we have synthesized two different ligands: one of which is an unsaturated macrocycle, and the other is a tripodal. Tren acts as a base for macrocycle formation and the pendent arm for the tripodal ligand. $\mathrm{Ni}(\mathrm{II}),{ }^{27} \mathrm{Zn}$ (II), and $\mathrm{Co}(\mathrm{III})$ metal ions do not form macrocycles. The macrocycle formation is unique for $\mathrm{Cu}(\mathrm{II})$. The plasticity of copper geometry probably can withstand the strain imposed by the saddlelike arrangement of this novel pyrazole-based macrocycle. In the Co(III) complex, the ligand is binegative, and only one type of complex is formed at different pHs whereas, in the cases of $\mathrm{Ni}$ (II) and $\mathrm{Zn}(\mathrm{II})$, the ligand acts as a neutral one.

Acknowledgment. Acknowledgment is made to the University Grants Commission, New Delhi, India, for awarding a minor research project in science [PSW-063/00-01(ERO)] to S. Paul. We thank Prof. K. Nag, Prof. Muktimoy Chaudhury of IACS, and Dr. K. Pramanik of Jogomaya Devi College, Calcutta, for many discussions.

Supporting Information Available: Crystallographic data in CIF format. This material is available free of charge via the Internet at http://pubs.acs.org.

IC0112185

(49) Gagné, R. R.; Koval, C. A.; Lisensky, G. C. Inorg. Chem. 1980, 19, 2584. 